\title{
The detection of subclinical leprosy using a monoclonal antibody based radioimmunoassay
}

\author{
M ASHWORTH, ${ }^{*}$ S SINHA, S A PATIL, G RAMU \\ \& U SENGUPTA $\dagger$ \\ Immunology Laboratory, Central JALMA Institute for Leprosy, \\ PO Box 31, Agra 282 001, India; *86 Cathles Road, London \\ $S W 129 L G$
}

Accepted for publication 12 January 1986

\begin{abstract}
Summary The monoclonal antibody based competition radioimmunoassay test was used to examine sera from 100 healthy household contacts of known leprosy patients. Only 6 out of 100 contacts had detectable specific antibodies.

It remains conjectural that this small fraction of contact subjects may be at much higher risk of developing disease than those without antibodies. Contacts who are antibody positive and lepromin negative (as were 4 of the 6), would best qualify for being offered chemoprophylaxis.
\end{abstract}

\section{Introduction}

Populations in endemic areas are exposed to the risk of developing leprosy because of delayed presentation of cases and prolonged infectivity before treatment is commenced. A large proportion, probably over $90 \%$ of those exposed will develop subclinical infection but only a much smaller proportion will subsequently develop clinical leprosy $;{ }^{2}$ the majority of subclinical infections are eradicated by an effective cell-mediated immune response to Mycobacterium leprae. A screening test, specific and predictive for leprosy is needed in order to reduce the large at-risk population to a small high-risk population which would be feasible to follow up with chemoprophylactic treatment.

Tests based on the cell-mediated response to $M$. leprae are not sufficiently specific because of cross-reactivity with $M$. tuberculosis and environmental mycobacteria. ${ }^{3}$ However, a new serological test for leprosy has been developed following the production of a monoclonal antibody, ML04, to the $35 \mathrm{~K}$ protein

$\dagger$ Reprints: Dr U Sengupta 
antigen of $M$. leprae. This was found to be fully specific for leprosy when tested against patients with active pulmonary tuberculosis, autoimmune diseases, carcinoma and healthy controls. ${ }^{5}$ Because of this high specificity this test was used to screen a population of healthy contacts of known leprosy source cases.

\section{Materials and methods}

One hundred household contacts of leprosy patients were studied. The contacts consisted of 84 relatives of patients attending the Central JALMA Institute for Leprosy, Agra and 16 relatives of patients attending the rural health centre at Deeg, Rajasthan. The contacts were all examined and excluded from the trial if they had any definite or probable clinical signs of leprosy. Each contact had a lepromin test performed and blood was taken for antibody titre determination. The diagnosis of leprosy and classification according to the Ridley-Jopling scale ${ }^{6}$ was made for each patient on the basis of clinical features, slit-skin smear and lepromin test in all cases and in addition histology of the skin lesion in 12 cases.

\section{LEPROMIN TEST}

Dharmendra lepromin ${ }^{7}$ was used to assess the skin delayed hypersensitivity to $M$. leprae in each contact. A positive early reaction (Fernandez reaction) was recorded after 48 hours if there was erythema and induration of $5 \mathrm{~mm}$ or more in diameter; a positive late reaction (Mitsuda reaction) was recorded at 4 weeks if there was a papule of $4 \mathrm{~mm}$ or more in diameter.

\section{SERUM ANTIBODY COMPETITION TEST (SACT)}

Antibodies to the $35 \mathrm{~K}$ antigen (MY2a epitope) of $M$. leprae were detected by radioimmunoassay. The principle of the test is competition between ${ }^{125} \mathrm{I}$ labelled $M$. leprae specific ML04 antibodies and homologous human antibodies present in the test serum for antigen binding. The technique followed was as described by Sinha et al. ${ }^{5}$ Results were expressed as the reciprocal of the serum dilution needed to inhibit $50 \%\left(\mathrm{ID}_{50}\right.$ value) of ${ }^{125} \mathrm{I}-\mathrm{ML} 04$ binding to the antigen.

\section{Results}

Six out of 100 household contacts of leprosy patients had antibodies to the MY2a epitope of $M$. leprae as determined by the SACT assay. All 6 SACT positive cases had low titres of antibody with an ID $_{50}$ value of 5. Figure 1 shows the pattern of inhibition of ${ }^{125}$ I-ML04 binding by the dilutions of the antibody positive sera. The hatched area indicates the range of inhibition by various control sera including those of tuberculosis patients. 


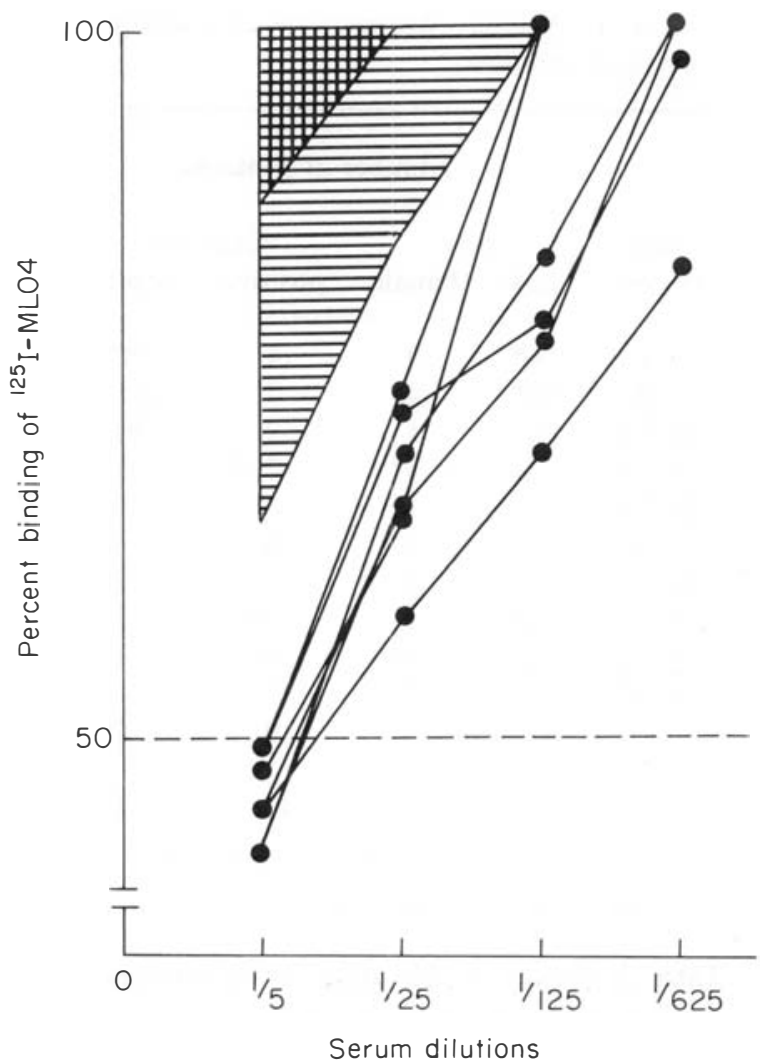

Figure 1. ${ }^{125} \mathrm{I}-\mathrm{ML} 04$ binding of diluted sera from the six positive contacts and from six healthy controls and six tuberculous patients. 四, healthy controls from endemic areas; 目, tuberculosis patients; - - contacts.

The age and sex distribution of the patients studied is shown in Table 1. Two of the $54(4 \%)$ contacts under 15 years old were antibody positive and 4 of $46(9 \%)$ contacts of 15 years and over were antibody positive. The early lepromin reaction was read in 91 cases and 21 of these returned after one month for reading of the late reaction. Nine cases were lost to follow up. Of the 6 SACT positive contacts 4 had a negative lepromin test (all Fernandez reaction) and 2 a positive test (one Fernandez reaction and one Mitsuda reaction) (Table 1). The duration that each contact was exposed to the source case is shown in Table 2. Only one out of the 31 $(3 \%)$ contacts exposed to LL cases was antibody positive whereas 4 out of 37 $(11 \%)$ contacts of BT cases were antibody positive. There was thus no positive correlation between the antibody positivity of contacts and exposure to a multibacillary source case. Only 2 out of the $24(8 \%)$ contacts exposed to a source case for 10 or more years was antibody positive whereas 4 of the $76(5 \%)$ contacts exposed for less than 10 years and 2 of the $42(5 \%)$ exposed for less than 5 years 
Table 1. Age/Sex distribution and lepromin status of contacts

\begin{tabular}{ccccc}
\hline & \multicolumn{4}{c}{ Number of contacts } \\
\cline { 2 - 5 } Age & \multicolumn{2}{c}{ Sex } & \multicolumn{2}{c}{ Lepromin } \\
(years) & male & female & positive & negative \\
\hline $0-4$ & $6^{*}$ & 9 & 4 & $10^{*}$ \\
$5-9$ & $16^{*}$ & 4 & 7 & $13^{*}$ \\
$10-14$ & 14 & 5 & 9 & 10 \\
$15-19$ & 7 & $3^{*}$ & 7 & $1^{*}$ \\
$20-24$ & 6 & 3 & 3 & 5 \\
$25-29$ & 4 & 1 & 0 & 3 \\
$30-34$ & 2 & 3 & 3 & 2 \\
$35-39$ & $4^{*}$ & 1 & $2^{*}$ & 3 \\
$40-44$ & 2 & $2^{*}$ & $3^{*}$ & 1 \\
$45-49$ & 2 & 2 & 2 & 0 \\
$50+$ & $3^{*}$ & 1 & 0 & $3^{*}$ \\
\hline Total & 66 & 34 & 40 & 51 \\
\hline
\end{tabular}

* One SACT positive contact

Table 2. Survey of the duration of exposure of contacts \& classification of source cases

\begin{tabular}{ccccccc}
\hline \multirow{2}{*}{$\begin{array}{c}\text { Duration of exposure } \\
\text { (years) }\end{array}$} & \multicolumn{4}{c}{ Number of tested contacts } \\
\cline { 2 - 6 } & TT $\dagger$ & BT & BB & BL & LL \\
\hline $0-4$ & 2 & $20^{*}$ & 7 & 6 & $7^{*}$ \\
$5-9$ & - & $10^{* *}$ & 5 & 7 & 12 \\
$10-14$ & - & 3 & 1 & $3^{*}$ & 8 \\
$15-19$ & - & $4^{*}$ & - & - & 1 \\
$20-24$ & - & - & - & 1 & 3 \\
\hline
\end{tabular}

* One SACT positive contact

$\dagger$ Classification of the source case.

were antibody positive. There was thus no positive correlation between antibody positivity and duration of exposure to a source case.

\section{Discussion}

Only a small proportion of those exposed to leprosy will go on to develop disease: 
the incidence of leprosy in a marriage partner of a patient is in the order of $5 \%{ }^{8} \mathrm{It}$ is therefore impractical to follow up and/or prophylactically treat all contacts of leprosy patients. However, preventative treatment could be considered if a small high-risk group could be identified. Only 6 out of 100 household contacts of leprosy patients were SACT positive. Antibodies detected by the fluorescence test were observed in a much larger proportion of contacts: one study ${ }^{9}$ found them in $81 \%$ (21 out of 26$)$ in a similar local population to that of this study and another ${ }^{1}$ found them in $92 \%$ ( 57 out of 62 ) of contacts in Japan. The MAB competition test thus defines a much smaller sub-group of all those with a subclinical infection.

Assuming that antibody positive contacts have a higher risk of subsequently developing leprosy than antibody negative contacts, this small fraction of all contacts could be carefully followed up. In this study 4 of the 6 antibody positive contacts had a negative lepromin test. It is accepted that cell-mediated immunity rather than antibodies can eliminate a subclinical infection. ${ }^{3}$ Thus the antibody positive contacts who lack a cell-mediated immune response to $M$. leprae as judged by a negative lepromin test would seem to carry the highest risk and could be considered for chemoprophylaxis.

It has been generally assumed that contacts exposed for prolonged periods to a multibacillary case and childhood contacts probably carry the highest risk. Although our study contained only 6 antibody positive contacts the obtained results did not support such trends. Two of the antibody positive contacts were relatives, husband and son, of a 39-year-old paucibacillary case (BT) and they had only been exposed to the overt disease for 3 years. The possibility remains that these two contacts were also exposed to another source as they lived in an area of moderate endemicity; the leprosy prevalence rate in the catchment area of this study is 8 per 1000 head of population. Childhood contacts were not more likely to be SACT positive than adults: $4 \%$ ( 2 out of 54 ) of those under 15 and $6 \%$ (1 out of 15) of those under 5 were seropositive. One study ${ }^{1}$ found fluorescent antibodies in higher titres in those under 5 years of age; the only positive contact under 5 in our study had a low titre of antibody as had all the other seropositive contacts.

In the previous study ${ }^{5}$ a higher overall antibody positivity $(30 \%)$ was reported among the contacts of leprosy patients. However, no information about the age and sex of these contacts as well as the endemicity of the area to which they belonged was recorded. It is possible that most of those contacts belonged to hyperendemic areas and were an older group with prolonged contact with the source cases. In the present study, only $9 \%$ positivity among the contacts above 15 years and $4 \%$ in those below 15 years of age was found.

Only a longitudinal prospective study could define the significance of antibody positivity by assessing the clinical outcome in the positive contacts and evaluating the predictive value of the MAB competition test. Preliminary results from a prospective study ${ }^{10}$ indicated that 6 out of 16 SACT positive contacts developed overt leprosy manifesting as multiple lesions of the BT/BB type within 
6 months of the serum being positive. Some of the contacts who were initially SACT positive became negative and developed a positive Mitsuda reaction. This ongoing survey suggests that those contacts who are ML04-SACT positive may be at a greater risk of developing clinical leprosy.

\section{Acknowledgments}

The authors are indebted to Dr R J W Rees of Clinical Research Centre, UK for the generous supply of soluble antigen of $M$. leprae from armadillo source. The authors are also grateful to Dr J Ivanyi of the MRC Tuberculosis and Related Infections Unit at Hammersmith Hospital for supplying monoclonal antibodies and for giving critical comments on the manuscript. Thanks are also due to the Indian Council of Medical Research, New Delhi, for financial assistance.

\section{References}

${ }^{1}$ Abe M, Minagawa F, Yoshino Y, Ozawa T, Saikawa K, Saito T. Fluorescent leprosy antibody absorption (FLA-ABS) test for detecting subclinical infection with Mycobacterium leprae. Int $J$ Lepr, 1980; 48: 109.

2 UNDP/World Bank/WHO. Special programme for research and training in tropical diseases. Sixth Programme Report. 1st July 1981 to 31 st December, 1982; Chapter 8.

${ }^{3}$ Godal T, Lofgran M, Negassi K. Immune response to M. leprae of healthy leprosy contacts. Int $J$ Lepr, 1972; 40: 243.

${ }^{4}$ Harboe M, Closs O, Bjune G, Kronvall G, Axelsen NH. Mycobacterium leprae specific antibodies detected by radioimmunoassay. Scand J Immunol, 1978; 7: 111.

${ }^{5}$ Sinha S, Sengupta U, Ramu G, Ivanyi J. Serological survey of leprosy and control subjects by a monoclonal antibody-based immunoassay. Int J Lepr, 1985; 53: 33.

${ }^{6}$ Ridley DS, Jopling WH. Classification of leprosy according to immunity. A five-group system. Int. J Lepr, 1966; 34: 255.

7 Sengupta U, Ramu G, Desikan K V. Assessment of Dharmendra antigen. II Standardisation of the antigen. Lepr India, 1979; 51: 316.

8 Mohamedali P. A study of conjugal leprosy. Int J Lepr, 1965; 33: 223.

9 Ramu G, Bharadwaj VP, Katoch K, Desikan KV. Studies of the healthy contacts of leprosy patients. Proceedings of the workshop on serological tests for detecting subclinical infection in leprosy. Sasakawa Memorial Health Foundation, 1983; 37: Tokyo.

${ }^{10}$ Ramu G, Malaviya GN, Bharadwaj VP, Sengupta U, Sinha S, Ramanathan VD, Pal C, Desikan KV. Studies on healthy contacts of leprosy patients - a preliminary report. Proceedings of the XII International Leprosy Congress, 1985. 